\section{D) Check for updates}

Cite this: Mater. Adv., 2021, 2, 2216

Received 22nd January 2021, Accepted 23rd February 2021

DOI: 10.1039/d1ma00053e

rsc.li/materials-advances

\title{
Anti-biofouling materials and surfaces based on mussel-inspired chemistry
}

\begin{abstract}
Sijia Li, Jingsi Chen, (D) Jingyi Wang and Hongbo Zeng (D)*
Mussel-inspired chemistry has attracted much research interest in manipulating material properties in surface engineering over the past decade due to its universality and versatility. To address biofouling issues, mussel-inspired molecular interaction strategies have provided promising methods for the design and development of anti-biofouling materials that can either repel proteins and microbes to avoid attachment or kill them in the surrounding environment. This review provides an overview of recent advances in mussel-inspired chemistry and its emerging applications in fabricating anti-biofouling platforms. The molecular interactions and adhesion mechanisms of mussel-inspired chemistry are first introduced. The applications of mussel-inspired chemistry in different anti-biofouling hydrogels, membranes and coatings are then discussed. Various bio-inspired material fabrication processes and surface functionalization strategies are presented, in which materials based on the mussel-inspired chemistry play different roles such as surface modifiers, intermediate layers or adhesive moieties to bind other components for functionalizing materials with anti-biofouling performance. Some remaining challenges are discussed, and future perspectives on mussel-inspired modification of anti-biofouling materials are also provided.
\end{abstract}

\section{Introduction}

Biofouling issues are generally initiated by the undesired adsorption and accumulation of proteins, bacteria, cells and other microorganisms on the surfaces of materials, which subsequently induce severe consequences such as infection in biosystems or

Department of Chemical and Materials Engineering, University of Alberta, Edmonton, ABT6G 1H9, Canada. E-mail: hongbo.zeng@ualberta.ca; Tel: +1-780-492-1044

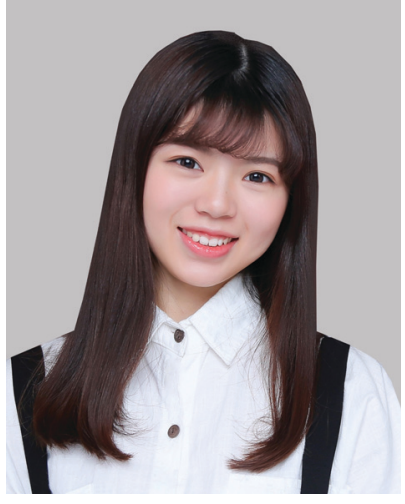

Sijia Li
Sijia Li is currently a PhD candidate at the University of Alberta under the supervision of Professor Hongbo Zeng. She received her BSc and MSC in Chemical Engineering from the Dalian University of Technology. Her research interests center on the development and application of bio-inspired interfacial materials with anti-fouling and anti-corrosion properties.

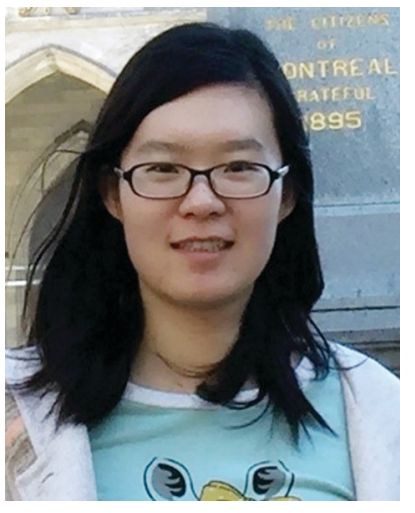

Jingsi Chen received her BSc in Materials Science and Engineering from Southeast University in 2012 and obtained a PhD degree in Materials Engineering from the University of Alberta in 2019. She is currently a post-doctoral fellow at the University of Alberta and her research mainly focuses on the development of functional supramolecular hydrogels toward bioengineering applications. 
passive (preventing adhesion) and active (detaching or degrading) strategies. ${ }^{5}$ In early studies, biocides were commonly employed to prevent bio-foulants from attaching to the surfaces. Researchers designed a variety of coatings which steadily release biocidal compounds such as antibiotics, silver particles or nitric oxides into the surrounding environments to effectively degrade or even kill the fouling organisms. ${ }^{6-8}$ However, due to the damage to the natural environment and human health, this type of material has been avoided, and other viable natural alternatives such as chitosan, ${ }^{9}$ capsaicin ${ }^{10}$ and enzymes ${ }^{11}$ are attracting increasing attention to improve this strategy. Another effective way to restrain the adsorption and attachment of foulants is modifying the surfaces with fouling-repellent coatings. For example, antibiofouling properties have been achieved through functionalizing substrate surfaces by hydrophilic polymers such as poly(ethylene glycol) (PEG) $)^{12-14}$ and zwitterionic molecules ${ }^{15-17}$ to resist protein and bacteria adsorption and reduce biofilm attachment. The key to their fouling-resistant properties is the steric exclusion effect and strongly hydrated charge groups on the hydrophilic polymer surfaces, which increases the energetic penalty of removing the surface hydration layer during the attachment of proteins and microorganisms. ${ }^{18}$

In recent years, natural creatures have inspired the development of numerous anti-biofouling platforms with outstanding and universal anti-biofouling performances. One novel strategy is based on the modulation of surface wettability mimicking the surface structures of biological materials. One of the most famous examples is the fabrication of superhydrophobic surfaces inspired by the self-cleaning lotus leaf, which significantly minimizes the contact between potential contaminants and the surfaces, thus inhibiting the adhesion of fouling organisms. ${ }^{19,20}$ Similarly, slippery liquid-infused porous surfaces (SLIPs) adopted by the Nepenthes pitcher plants have also been developed and shown powerful omniphobic capability to resist fouling liquids or organisms at smooth liquid-like interfaces. ${ }^{21,22}$

In addition to the bio-inspired designs of nanostructures, the multiple functionalities of natural components can also be mimicked and utilized to functionalize material surfaces. ${ }^{23-25}$ Lately, anti-biofouling materials inspired by the adhesive secretions of marine mussels have attracted considerable interest. ${ }^{26,27}$ The mussel foot proteins (Mfps) secreted by mussels could form a strongly adhesive interaction with various substrates in a wet environment, allowing the mussels to adhere on almost all types of substrates. It has been reported that 3,4-dihydroxyphenylalanine (DOPA), a critical functional component in Mfps, plays a critical role in the versatile adhesion properties of marine mussels. Various molecular interactions are detected in DOPA and its derivatives such as dopamine and polydopamine (PDA), which are commonly referred to as "DOPA chemistry" and have sprouted the development of many mussel-inspired materials such as functional coatings and hydrogels with diverse properties. ${ }^{28,29}$ Mussel-inspired chemistry has been widely implemented in surface and interface engineering for multipurpose functionalization to construct superior interfaces, especially in aqueous

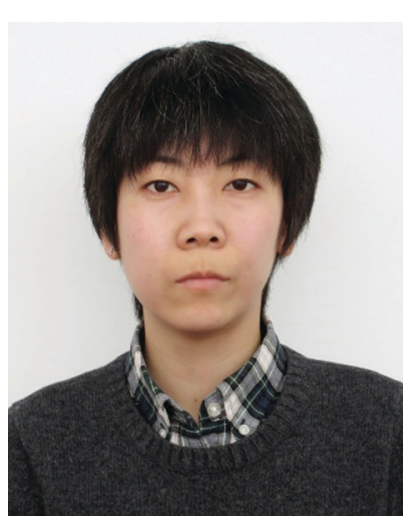

Jingyi Wang
Jingyi Wang is a Research Associate in the Department of Chemical and Materials Engineering at the University of Alberta. She received her BSc degree from the Wuhan University of Technology, and $\mathrm{PhD}$ degree in 2011 from Beihang University. Her research interests are interfacial interactions in colloidal systems, surface characterization of materials, and application of electrochemistry in the study of interfacial properties.

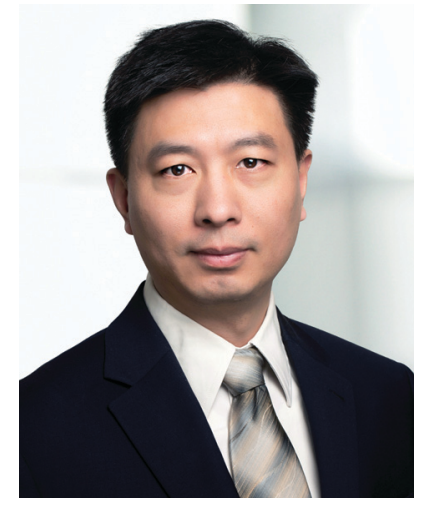

Hongbo Zeng
Hongbo Zeng is a Professor in the Department of Chemical and Materials Engineering at the University of Alberta, a Tier 1 Canada Research Chair in intermolecular forces and interfacial science, a Fellow of the Canadian Academy of Engineering and a Member of the Royal Society of Canada's College of New Scholars. He received his BSc and MSc degrees from Tsinghua University, and obtained his PhD in chemical engineering at the University of California, Santa Barbara, under the supervision of Prof. Jacob Israelachvili and Prof. Matthew Tirrell. His research interests are colloid and interface science, and functional materials, with a special focus on intermolecular and surface interactions in soft matter and engineering applications. He has published about 350 refereed research journal articles. His research has been recognized by many awards such as the Petro-Canada Young Innovator Award, Martha Cook Piper Research Prize, The Canadian Journal of Chemical Engineering Lectureship Award, CSChE Innovation Award of the Chemical Institute of Canada, International Award for Outstanding Young Chemical Engineer, and the NSERC E. W. R. Steacie Memorial Fellowship. 
environments. ${ }^{5,30}$ In the anti-biofouling area, much effort has been dedicated to the preparation of anti-biofouling materials through DOPA chemistry-mediated cohesion and adhesion mechanisms. ${ }^{31-33}$ Dopamine has the advantage of deposition and self-polymerization over various inorganic and organic substrates by forming PDA layers under mild alkaline conditions. ${ }^{26}$ The highly hydrophilic PDA layers with intrinsic fouling resistance properties $^{34,35}$ and intrinsic antimicrobial activity ${ }^{35,36}$ behave as protective coatings to mitigate the fouling caused by proteins and microorganisms. Moreover, the catechol-containing molecules can react with various functional groups as adhesive agents due to the versatile adhesion derived from DOPA chemistry, constructing many inorganic/organic composite materials with excellent antibiofouling performance via surface modification or direct assembly. ${ }^{37-40}$ During the preparation process of diverse antibiofouling materials, materials based on mussel-inspired chemistry could play different roles such as surface modifiers, intermediate layers or adhesive moieties to bind other components for functionalizing materials with anti-biofouling performance (Fig. 1).

This review first summarizes the various molecular interactions and interaction mechanisms involved in mussel-inspired chemistry, followed by functionalization mechanisms using DOPA chemistry to modify different surfaces and polymers. Then, the recent progress in the design of novel anti-biofouling materials derived from mussel-inspired chemistry is discussed, including hydrogels, membranes, and coatings. Meanwhile, the roles dopamine/PDA play in the fabrication of anti-biofouling materials and the associated preparation strategies are presented. The remaining challenges and future perspectives on the further development and applications of mussel-inspired anti-biofouling materials are also discussed.

\section{Mussel adhesion mechanisms}

Mussels tether themselves to diverse surfaces underwater such as rocks, metal, wood and even some well-known low-fouling materials like Teflon by the secreted adhesive foot proteins, via the formation of mussel byssus consisting of adhesive thread and plaque. ${ }^{41-43}$ Upon the immobilization of the mussel foot to the desired attachment location, Mfps can form an adhesive plaque that securely anchors the mussel to the substrate surface even under waves with high shear forces. It is reported that the byssus consists of over twenty different known protein components, in which the six types of important Mfps namely Mfp 1-6 have been well investigated and considered to have played critical roles in mussel adhesion. A schematic for the distribution of Mfp 1-6 confined to the byssal thread and plaque is shown in Fig. 2a and b. ${ }^{44} \mathrm{Mfp}-1$ is the first identified phenolic protein with a large molecular weight of $\sim 108 \mathrm{kDa}$, which forms protective and stiff cuticles covering the byssal threads and plaques via oxidation or metal ion-mediated

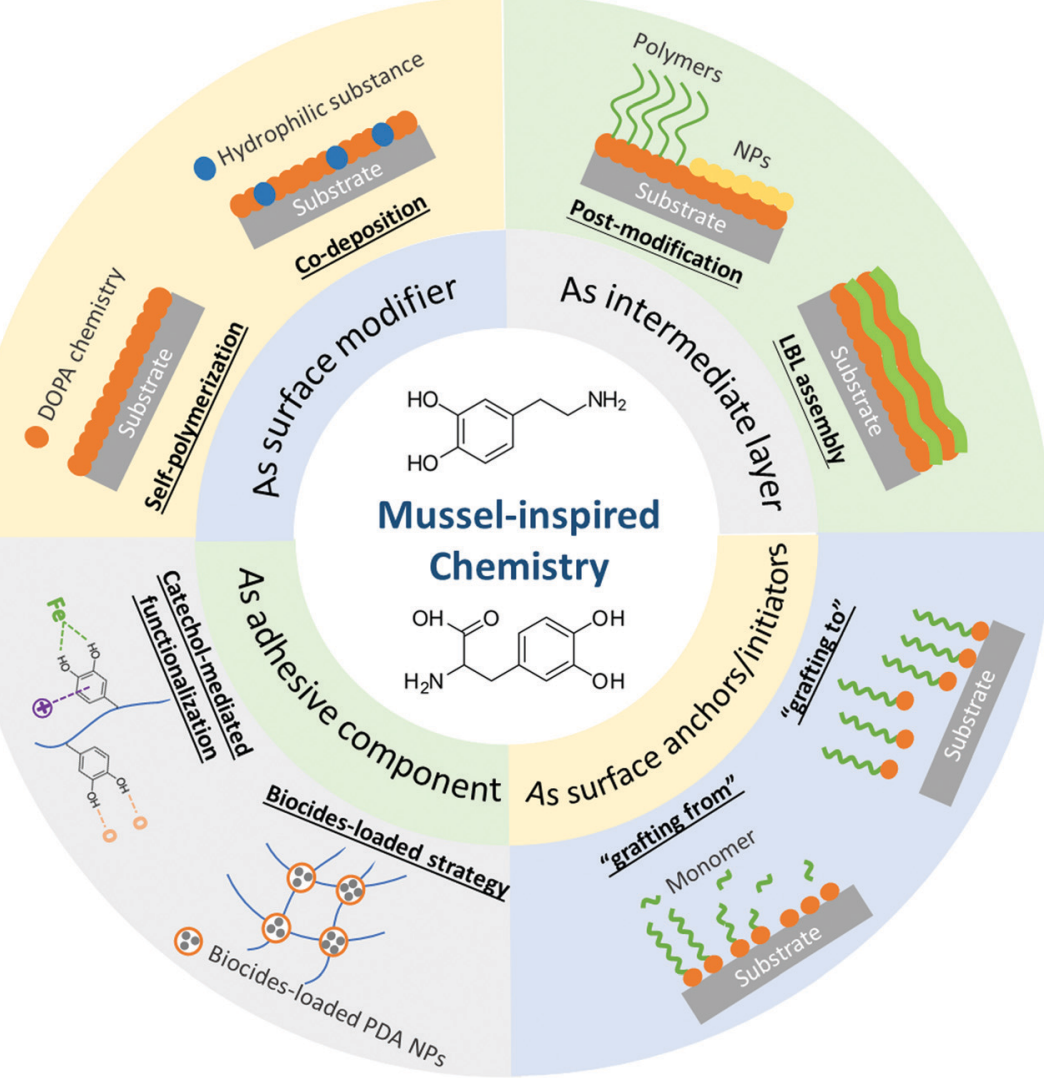

Fig. 1 The roles of mussel-inspired chemistry in the fabrication of anti-biofouling materials and the associated preparation strategies. 
(a)

(b)
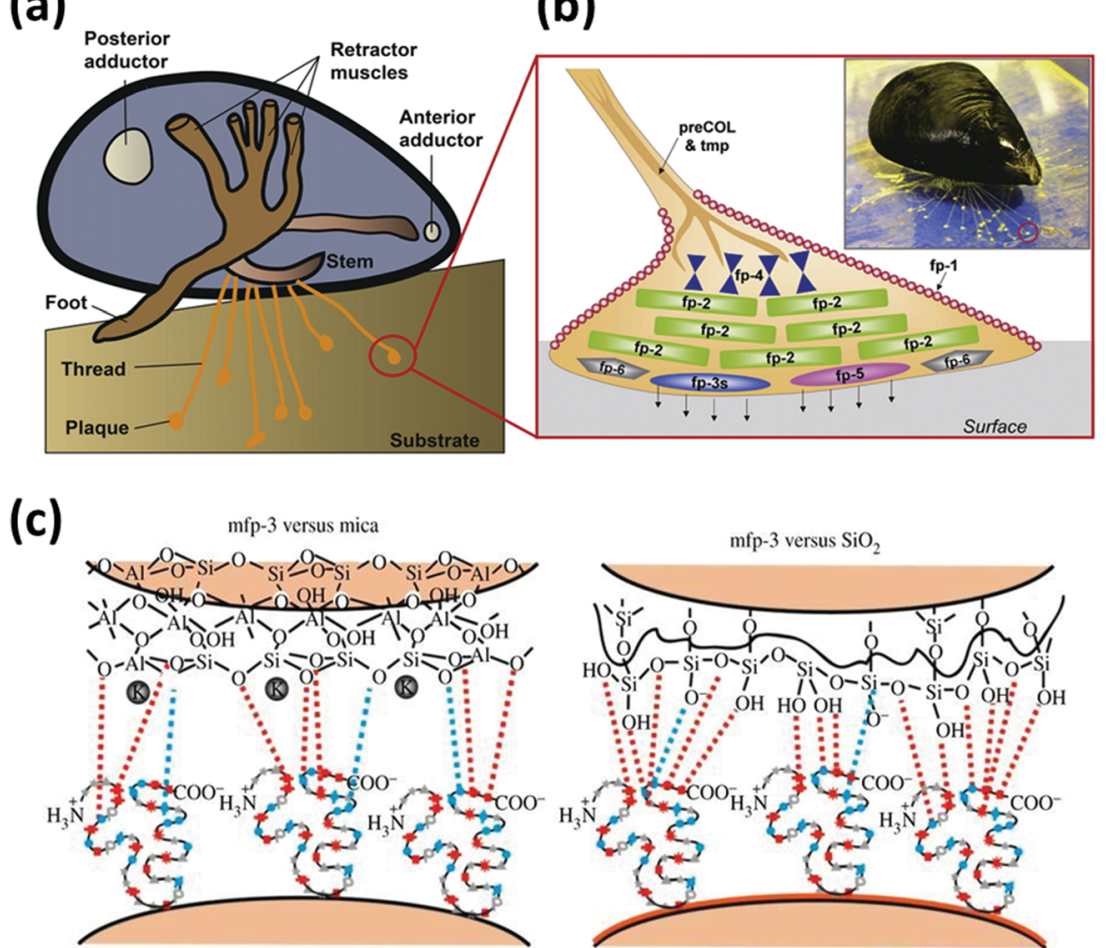

mfp-3 versus PMMA
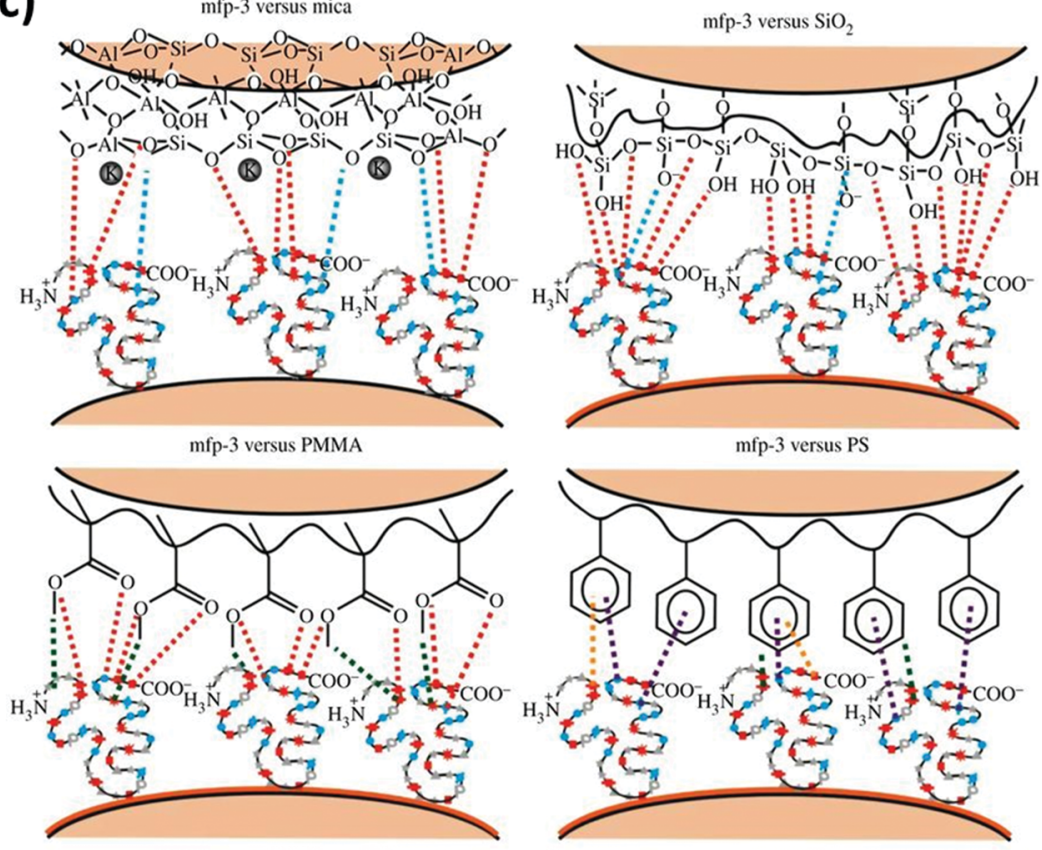

mfp-3 versus PS

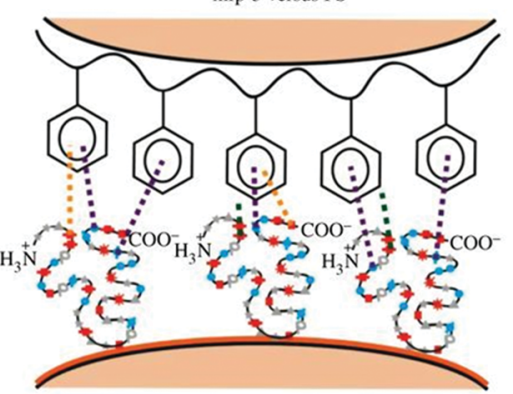

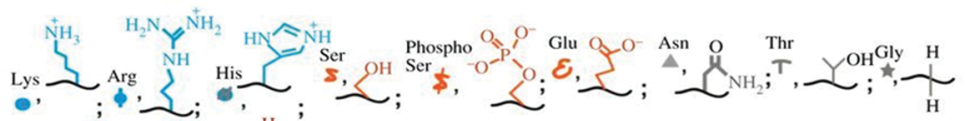

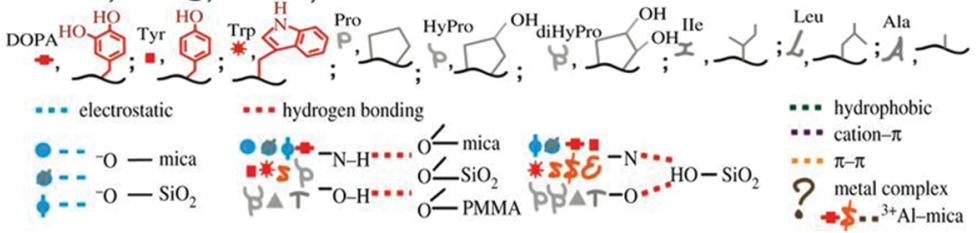

Fig. 2 (a) Schematic of a marine mussel that attaches to a substrate via byssal threads underwater. (b) Schematic of an enlarged byssal plaque (red circle in (a)) which illustrates the approximate distribution of various mussel foot proteins (Mfps), with the inset showing a marine mussel attached on a mica sheet. Reproduced with permission. ${ }^{44}$ Copyright 2010, The American Society for Biochemistry and Molecular Biology. (c) Illustration of interaction mechanisms between Mfp-3 and four different surfaces (i.e., mica, $\mathrm{SiO}_{2}$, polymethyl methacrylate (PMMA) and polystyrene (PS)). Reproduced with permission. ${ }^{41}$ Copyright 2013, The Royal Society.

crosslinking. ${ }^{45,46}$ Mfp-2 is the most abundant structural and cohesive component located in the inner part of the plaque, contributing to $25-40 \%$ of the plaque proteins. ${ }^{47,48} \mathrm{Mfp}-3$ and Mfp-5 are adhesive proteins with relatively small molecular mass and different variants located at the interface between the plaque and substrates, both of which are considered to act as important interfacial primers for mussel adhesion. ${ }^{49-52}$ Mfp-4 is proposed to serve as a coupling agent at the thread-plaque junction to connect the pre-polymerized collagens (preCOLs) in the inner core of the byssal thread and Mfp- 2 in the plaque. ${ }^{53,54}$ Mfp- 6 contains cysteine thiols, which create an environment for the redox reaction of the adhesive substances in the proteins. ${ }^{55}$

Previous studies have shown that the six mussel adhesive proteins in the byssus possess repetitive decapeptide sequences 
with a high DOPA content, demonstrated to mediate their high adhesion strength. ${ }^{56,57}$ In particular, Mfp-3 and Mfp-5 found at the adhesive interface contain the highest content of DOPA residues (25-30 mol\%) than other Mfps, indicating that DOPA plays a key role in achieving remarkable adhesion of mussel to diverse surfaces. ${ }^{49,52}$ Various fundamental studies using nanomechanical techniques such as a surface force apparatus (SFA) and an atomic force microscope (AFM) were conducted to investigate the interaction mechanisms of Mfps as well as single DOPA moieties to various substrates. For example, Lu et al. investigated and compared the molecular interaction forces between different Mfps (Mfp-1, Mfp-3, and Mfp-5) and various surface chemistries by SFA, as shown in Fig. 2 c. $^{41}$ All Mfps showed adhesive versatility to both hydrophilic and hydrophobic substrates. It was reported that for hydrophilic surfaces like mica, the protein-surface adhesion mainly resulted from hydrogen bonding interactions between the phenolic hydroxyl group and hydrogen-bonding acceptors, as well as the formation of covalent bonds. However, hydrophobic or $\pi-\pi$ interactions played a crucial role when catechol contacted non-polar polymers such as polystyrene (PS). The wet adhesion strength of Mfps to substrates depended on various factors such as the interplay of different amino acids, protein molecular weights and protein chain flexibility. Among them, the interactions of DOPA are one of the most essential contributors to Mfps adhesion to different surfaces. The interaction forces of a single DOPA moiety with substrates such as Ti were characterized by single molecule force spectroscopy experiments using AFM, improving the fundamental understanding of adhesion between DOPA units and substrates in water. ${ }^{58}$ Various interactions including hydrogen bonding, metal-catechol coordination, electrostatic, cation $-\pi$, anion $-\pi$ and $\pi-\pi$ aromatic interactions have been demonstrated to contribute to the versatile DOPA-mediated underwater adhesion to different substrates. $^{44,59-65}$

To date, attributed to the strong and universal adhesion ability of catechol structure, DOPA chemistry, occurred in DOPA as well as its derivatives such as dopamine and PDA, has been employed for wide applications such as surface modification and preparation of medical adhesives and biomaterials. In synthetic materials, the catechol structure of DOPA is often used for both adhesion (binding to substrates) and cohesion (crosslinking of the adhesive material) (Fig. 3) ${ }^{66,67}$ Several strategies have been used for oxidizing or crosslinking DOPA to form covalently grafted functional layers, including reacting DOPA with various functional groups (e.g., thiols, amines) via the Michael addition or Schiff base reaction. ${ }^{40,68}$ Besides, the redox transition between catechol and quinone under mild conditions enables facile grafting and anchoring of polymer chains onto the catechol-modified surfaces through surface-initiated atom transfer radical polymerization (SI-ATRP) and reversible addition fragmentation transfer polymerization

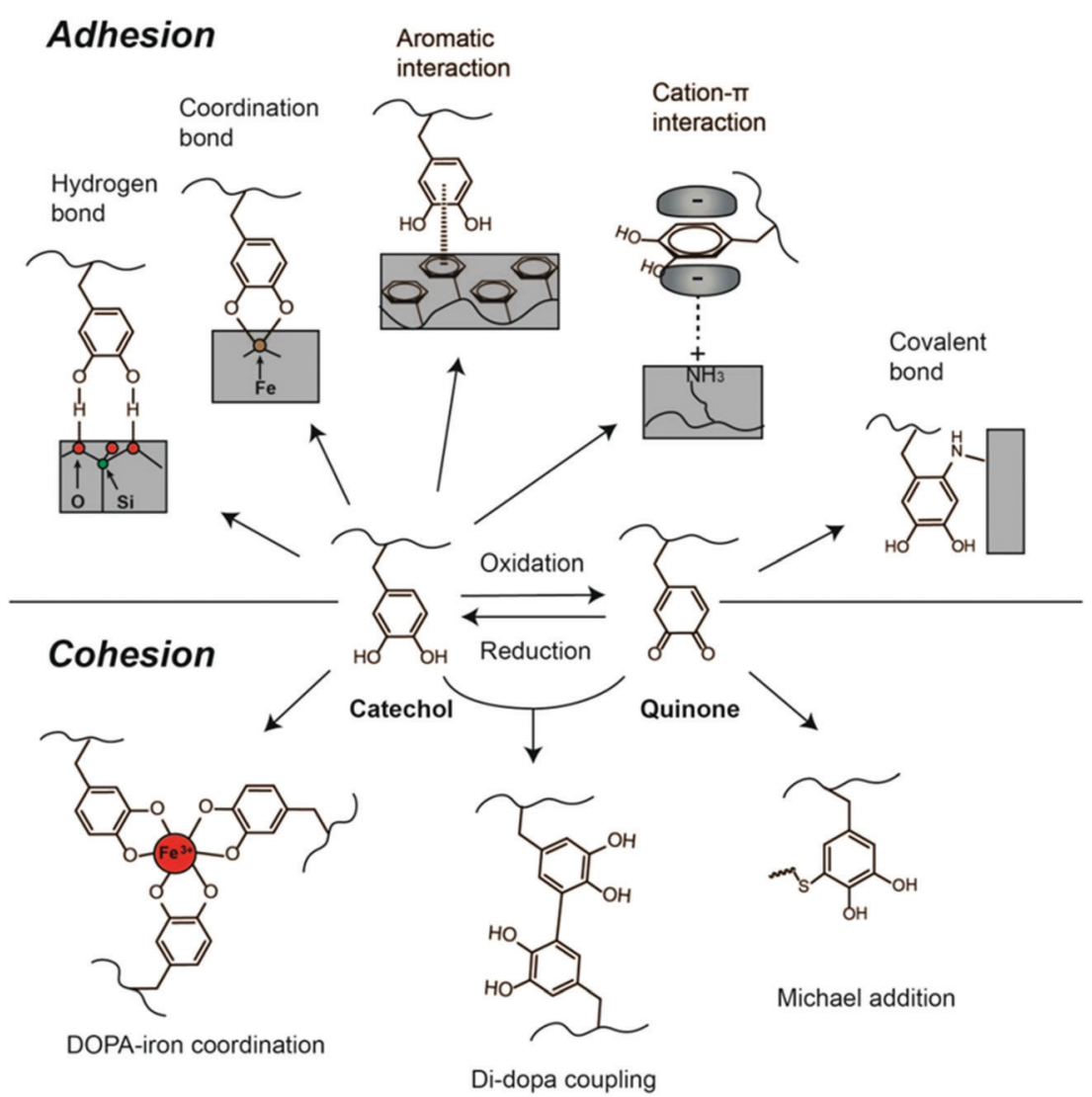

Fig. 3 DOPA-mediated reaction chemistry for adhesion and cohesion interactions. Reproduced with permission. ${ }^{33}$ Copyright 2016, Elsevier. 
(RAFT). ${ }^{69,70}$ Furthermore, the catechol and amino groups could also serve as nucleation sites to chelate metal ions, which usually act as the crosslinking points in the metal ion-bridged polymers and hydrogels. ${ }^{71,72}$ For example, DOPA can form various complexes with $\mathrm{Fe}^{3+}$, including bis- and tris-complexes of DOPA-Fe ${ }^{3+}$ coordination under an alkaline environment, leading to strong cohesion of polymers, which indicates that catechols can interact with $\mathrm{Fe}^{3+}$ to form $\mathrm{pH}$-dependent reversible crosslinking of materials. ${ }^{73,74}$

Moreover, the catechol structure can form crosslinking networks via autoxidation and self-polymerization in alkali solutions, forming a PDA thin film attached to the substrates. ${ }^{68,75}$ To date, there have been no final conclusions on the adhesion mechanisms and structures of the PDA yet. Several studies suggested that PDA is a supramolecular aggregate consisting of 5,6-dihydroxyindoline and its derivatives formed by charge transfer, $\pi-\pi$ stacking and hydrogen bonding. ${ }^{76,77}$ Similar to DOPA, PDA contains active groups including catechol and amine groups and can also trigger interfacial interactions with other functional groups. The DOPA-mediated adhesion and cohesion mechanisms have cultivated feasible and simple strategies for biomaterial synthesis and functionalization. The various physical and chemical interactions offered by DOPA chemistry have motivated the development of many functional materials.

\section{Mussel-inspired anti-biofouling materials}

\subsection{Mussel-inspired anti-biofouling hydrogels}

The mussel-inspired molecular interaction strategy also provides a promising route to enable the introduction of functional components and incorporation of anti-biofouling functionality into hydrogel materials. The catechol group is considered to possess inherent antibacterial properties when introduced into hydrogel networks. It is reported that during the occurrence of the catechol oxidation process in catechol-modified hydrogels or microgels, reactive oxygen species (ROS) such as superoxide and $\mathrm{H}_{2} \mathrm{O}_{2}$ are often generated as secondary products, which could completely prevent colony formation of bacteria such as $E$. coli and effectively inactivate the infectivity of diverse types of viruses. ${ }^{78-80}$ In addition, amine groups could be protonated at physiological $\mathrm{pH}(\sim 7.4)$, which would adsorb the negatively charged bacteria through electrostatic interactions, leading to the resistance to bacterial growth. Previous studies showed that the excellent antibacterial properties of biomaterials with a high PDA content could be attributed to the rich amino content in the PDA molecules, which were positively charged under neutral $\mathrm{pH}$ conditions and could interact with negatively charged components on the bacterial cells, causing disruption and lysis of the cells. $^{81,82}$

To enhance the anti-biofouling or antibacterial properties, hydrogels are also loaded with biocides such as antibiotics, bactericidal agents and antibacterial polymers besides catecholcontaining moieties. Gao et al. prepared an injectable hydrogel by mixing ciprofloxacin (Cip, a potent antibiotic)-loaded PDA nanoparticles (NPs) and glycol chitosan (GC), exhibiting antibacterial effects for both Gram-negative and Gram-positive bacteria (Fig. 4a). ${ }^{83}$ The commonly used antibiotic Cip was encapsulated in PDA NPs through $\pi-\pi$ stacking and hydrogen bonding to form PDA NP-Cip complexes, which then acted as building blocks to crosslink amine-rich GC through the Schiff base reaction and Michael addition, yielding an injectable hydrogel with outstanding antibacterial activity. Besides, noble metal NPs with strong inhibitory and bactericidal effects can also be introduced in hydrogel networks for preparing anti-biofouling hydrogels. The metal ions are often sequestered into the hydrogel network through catecholmetal chelation interactions, which can interfere with functions of bacterial cells via a competitive protein complexation mechanism. ${ }^{84,85}$ Messersmith and coworkers developed a silver-releasing antibacterial hydrogel through a spontaneous redox reaction between branched catechol-derived PEG and $\mathrm{Ag}^{+} \cdot{ }^{86} \mathrm{Ag}^{+}$was employed to induce the crosslinking of the hydrogel and reduced to Ag NPs via catechol oxidation, endowing the formed Ag NPs-filled hydrogel with antibacterial activity. Combining the antifouling properties of PEG, the hydrogels were found to inhibit bacterial growth and resist bacterial and mammalian cell attachment.

Another strategy to endow the hydrogels with anti-biofouling and antimicrobial effect is incorporating weak organic acids (e.g., citric acid) with a high content of carboxyl moieties into the hydrogel structures. The abundant free carboxyl groups in citrate-based polymers can lower the local $\mathrm{pH}$, suppress the nicotinamide adenine dinucleotide (NADH) oxidation in cell metabolism, and chelate the metal ions in the cell wall, which affects the cell permeability for nutrient uptakes, causing damage and death of bacterial cells. ${ }^{88}$ Guo et al. prepared an undecylenate citric acid-containing catechol-citrate-PEG hydrogel by introducing the anti-fungal agent 10-undecylenic acid (UA) into the citrate-based mussel-inspired adhesives. ${ }^{89}$ The combination of UA and citrate-based polymers led to dual-functional anti-bacterial and anti-fungal performances of the hydrogel, which showed resistance against human-derived mesenchymal stem cells and fungal as well as bacterial inhibition against C. albicans (fungi), S. aureus (Gram-positive bacteria) and E. coli (Gram-negative bacteria).

Introducing hydrophilic macromolecules into the hydrogel network to construct a hydrophilic layer on the surface is another widely employed strategy for anti-biofouling hydrogel preparation. The grafted hydrophilic polymers such as acid betaine (PSBMA), hyaluronic acid (HA) and PEG in the hydrogel structures can strongly couple water molecules, acting as a natural barrier to the adhesion of proteins and microorganisms. Our group fabricated an injectable hydrogel based on the self-assembly of an ABA triblock copolymer poly[( $N$-isopropylacrylamide $)-c o-(N-3,4-$ dihydroxyphenethyl acrylamide $)]-b$-poly(ethylene oxide)- $b$-poly $[(N$ isopropylacrylamide)-co-( $N$-3,4-dihydroxyphenethyl acrylamide)] (DNODN), which contained catechol-functionalized poly $(\mathrm{N}-$ isopropylacrylamide) (PNIPAM) as the thermo-sensitive terminal block and poly(ethylene oxide) (PEO) as the hydrophilic mid-block of the copolymer (Fig. 4b). ${ }^{87}$ The polymer was synthesized through 
(a)
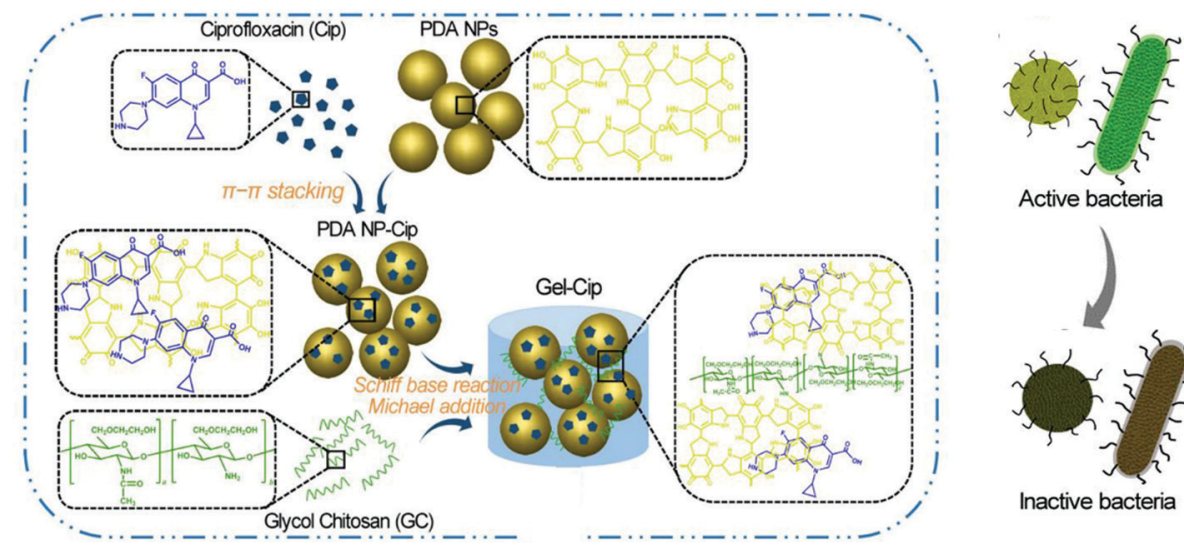

Active bacteria

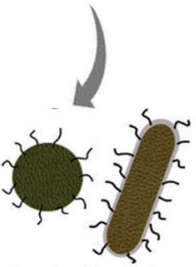

Inactive bacteria

(b)

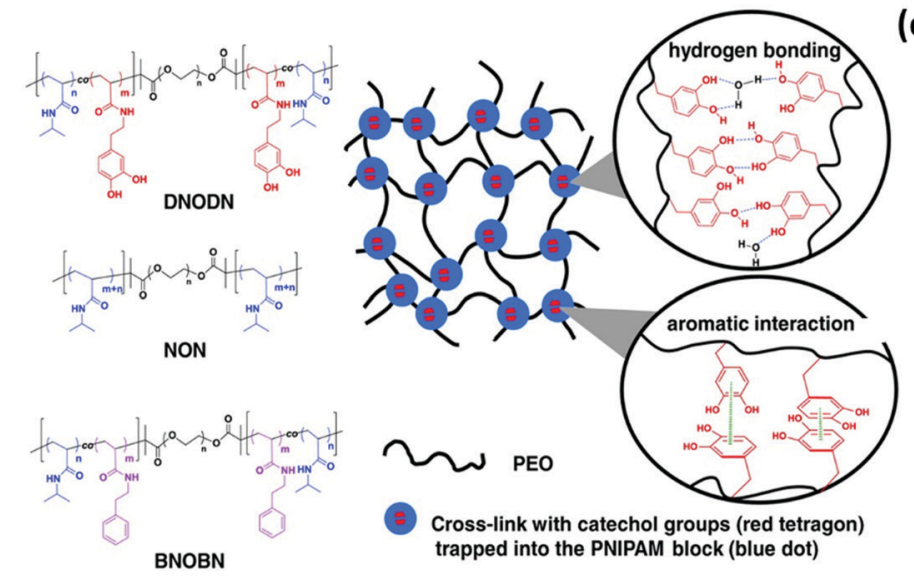

(c)
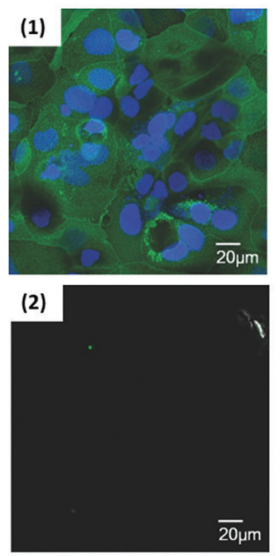

(d)

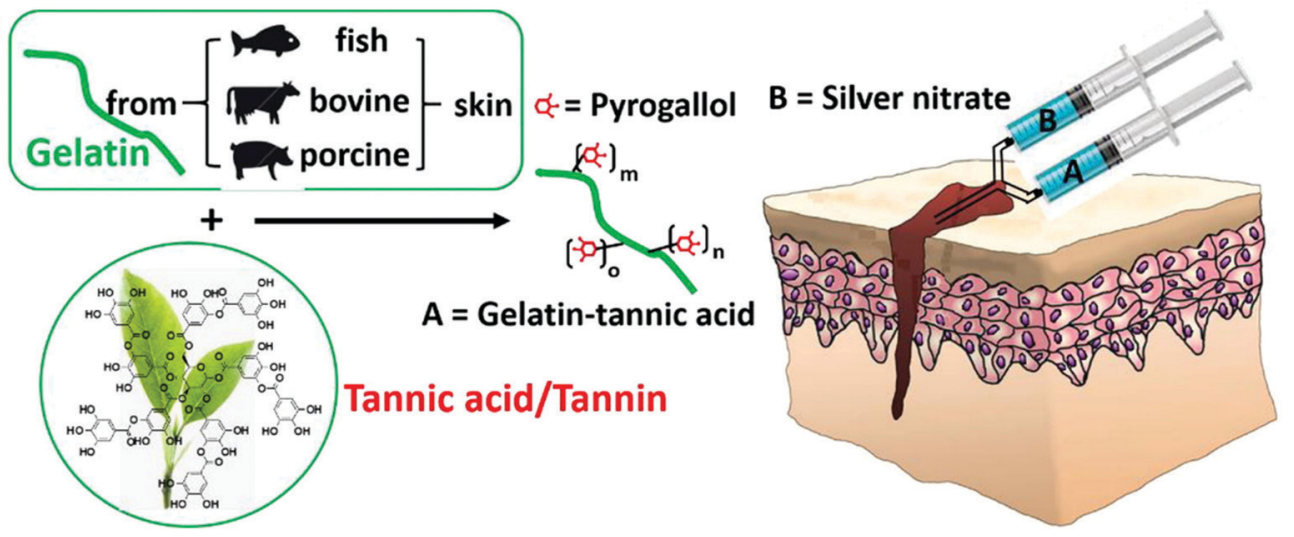

Fig. 4 (a) Schematics of the synthetic route of Gel-Cip and NIR light irradiation-triggered Cip release from Gel-Cip for bacterial inactivation. Reproduced with permission. ${ }^{83}$ Copyright 2019, Elsevier. (b) Chemical structure of the ABA tri-block copolymers DNODN, NON and BNOBN. Schematic of a proposed structure of the DNODN hydrogel. (c) Representative fluorescence microscopy images of (1) uncoated and (2) DNODN hydrogel coated microwell dishes after exposure to Caco-2 cells for $48 \mathrm{~h}$. Reproduced with permission. ${ }^{87}$ Copyright 2015, Wiley-VCH. (d) The bulk network and tissue bonding structures of the tannin-inspired antimicrobial bioadhesives crosslinked by silver nitrate (SN). Reproduced with permission. ${ }^{85}$ Copyright 2018 , Elsevier.

the aggregation of PNIPAM as well as the mussel-inspired noncovalent interactions including hydrogen bonding and aromatic interactions between catechol moieties. The DNODN hydrogel exhibited an excellent anti-biofouling performance by effectively preventing the attachment of human colorectal cancer (Caco-2) cells, which was attributed to the anti-biofouling properties of the hydrophilic PEO (Fig. 4c). Similarly, an injectable hydrogel with both anti-biofouling and antibacterial properties was designed by synthesizing an ABA triblock copolymer comprising a catecholfunctionalized PEG-based A block and a quaternized B block (poly([2-(methacryloyloxy)-ethyl] trimethyl ammonium iodide)), PMETA). ${ }^{90}$ The PEG-PMETA hydrogel showed inhibition to the adhesion of Caco-2 cells and exhibited lethality to E. coli. The B block was permanently hydrophilic, so the hydrogel molecules 
were highly hydrated, resulting in the resistance to the attachment of nonspecific proteins. Besides, the cationic trimethylammonium side chains in the hydrogel structure killed bacteria through the disruption of the negatively charged cell membrane. Compared with the anti-biofouling hydrogels containing metal NPs and metal ions through mussel-inspired catechol-metal coordination, incorporating hydrophilic polymers to the hydrogel networks via catechol-mediated hydrogen bonding and $\pi-\pi$ interactions exhibits great superiority in bioengineering applications, due to the reduced cytotoxicity and enhanced transparency in the metal-free environment of the hydrogels.

Recently, phenols and polyphenols have attracted growing attention due to their similar structure to dopamine. For example, tannic acid (TA), as the most studied polyphenol, was proven to participate in diverse bonding functionalities and oxidation-induced polymerization through multiple noncovalent interactions similar to catechol, which enables it to functionalize materials as an adhesive agent with additional antibacterial properties. The possible antibacterial mechanism of the phytochemical tannic acid has been proposed, which could interact with the proteins in the bacterial cell walls and act as an alternative target that results in cell membrane disruption and bacteria lysis. Guo et al. developed tannin-inspired anti-biofouling adhesives by one-step Michael addition of tannic acid and gelatin under oxidizing conditions, which showed bacterial inhibition ratios of nearly $100 \%$ against both $S$. aureus and $E$. coli and effectively killed fungal C. albicans (Fig. 4d). ${ }^{85}$

\subsection{Mussel-inspired anti-biofouling surfaces}

3.2.1. Mussel-inspired anti-biofouling membranes. The surface engineering of membranes has attracted much attention since a new generation of polymer membranes is required to meet the needs of both high permeation performance and multifunctional properties. The water permeation performance and biofouling resistance properties are usually determined by the membrane surface properties including wettability, charge, surface chemistry, roughness, etc.

In general, compared with hydrophobic surfaces, hydrophilic surfaces show better wettability and biofouling resistance, which increases water permeation flux and limits foulant adsorption on the surface and pores of the membrane. However, as many commercial polymer membranes are made of hydrophobic materials, surface biofouling becomes one of the most crucial problems during the filtration process. To improve the performance of membranes, membrane hydrophilization strategies have been motivated and exploited to construct anti-biofouling surfaces. Dopamine has been proven as an ideal candidate to minimize membrane biofouling, which deposits and forms a self-polymerized PDA coating on the membrane surfaces under basic conditions. PDA strongly attaches to various substrates and displays high hydrophilicity because of the presence of many hydrophilic functional groups such as carboxy, amino, imine and phenol groups. Dopamine deposition is generally a stable and controllable process, which can be adjusted by changing $\mathrm{pH}$, dopamine concentration, deposition time and atmosphere. Zhu and coworkers reported dopamine as hydrophilic modifiers for hydrophobic polymer membranes. ${ }^{91}$ Dopamine was oxidized and self-polymerized, which generated hydrophilic PDA coating on the membrane surface, leading to a $20-30 \%$ decrease in the water contact angles of hydrophobic membranes including polytetrafluoroethylene (PTFE), polyethylene (PE), polyvinylidene fluoride (PVDF). According to the reports, the water contact angles of membranes after PDA deposition generally reduce to the range of $37-90^{\circ}$, depending on the inherent wettability of original membranes. ${ }^{92}$ In addition, some DOPA-derivatives such as PDA and poly(3-(3,4-dihydroxyphenyl)-L-alanine (L-DOPA)) molecules are proven to exhibit zwitterionic properties due to the presence of diverse functional groups containing both positively charged (amino group) and negatively charged (phenolic hydroxyl and carboxylic acid) groups. ${ }^{93,94}$ For example, the isoelectric point of PDA is determined to be around $\mathrm{pH}$ 4. At high $\mathrm{pH}$, it exhibits a net negative charge because of the deprotonation of the phenolic groups, whereas at $\mathrm{pH}$ values below the isoelectric point, the amino groups will be protonated, leading to a positively charged PDA layer on the membrane surface. ${ }^{95}$ In this case, the membrane surfaces could be modified by the deposition of PDA or poly(L-DOPA) films with zwitterionic properties to enhance the anti-biofouling performance. Zou and coworkers applied a self-polymerized L-DOPA film to form a zwitterionic coating on reverse osmosis (RO) membranes to alter their surface properties for biofouling-resistant purposes, where the coated membranes showed an increase of water permeability and improved fouling resistance to the proteins and organic foulants. ${ }^{94,96}$

However, for the pure PDA-coated membranes, the presence of aromatic rings would limit the hydrophilicity in the PDA coating, and the pores of membranes could also be easily blocked by PDA aggregates, which limit the permeation and anti-biofouling performance of modified membranes. To address these problems, a one-step co-deposition approach has been developed to introduce other hydrophilic species into PDA layers to further improve the hydrophilicity and anti-biofouling properties of membranes. Chen et al. constructed a composite layer by co-deposition of dopamine and non-ionic polymers such as polyvinyl alcohol (PVA) and PEG onto PVDF membranes (Fig. 5a). ${ }^{97}$ The results suggested that the hydrophilicity and surface free energy of all modified membranes were significantly enhanced, which improved the hydration capability, oil/water separability and protein fouling resistance. In addition, dopamine was also reported to co-deposit with polyethyleneimine (PEI) to functionalize membranes. ${ }^{98}$ Dopamine could react with PEI via the Schiff base or Michael addition reaction, which effectively inhibits the non-covalent interactions among dopamine molecules, thus reducing the PDA aggregation. As an amino-rich polymer, the interposition of PEI also provides hydrophilic groups for further hydrophilization. The addition of PEI significantly decreased the surface roughness of PDA coatings, thus improving water flux and reducing membrane fouling. Likewise, Zhang et al. modified polyacrylonitrile (PAN) membranes by the co-deposition of PDA, PDA/PEI, PDA $/ \mathrm{Co}^{2+}$, and PDA/PEI $/ \mathrm{Co}^{2+}$, which resulted in water contact angles of $55^{\circ}, 41^{\circ}, 27^{\circ}$, and $18^{\circ}$, respectively. ${ }^{99}$ As shown in Fig. 5b, during the coating process, dopamine 
(a)

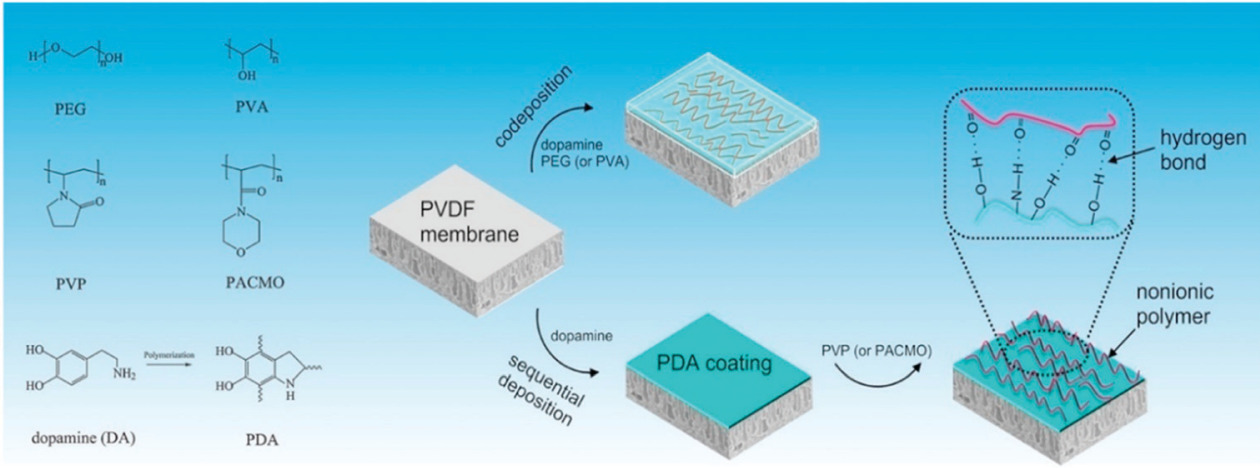

(b)

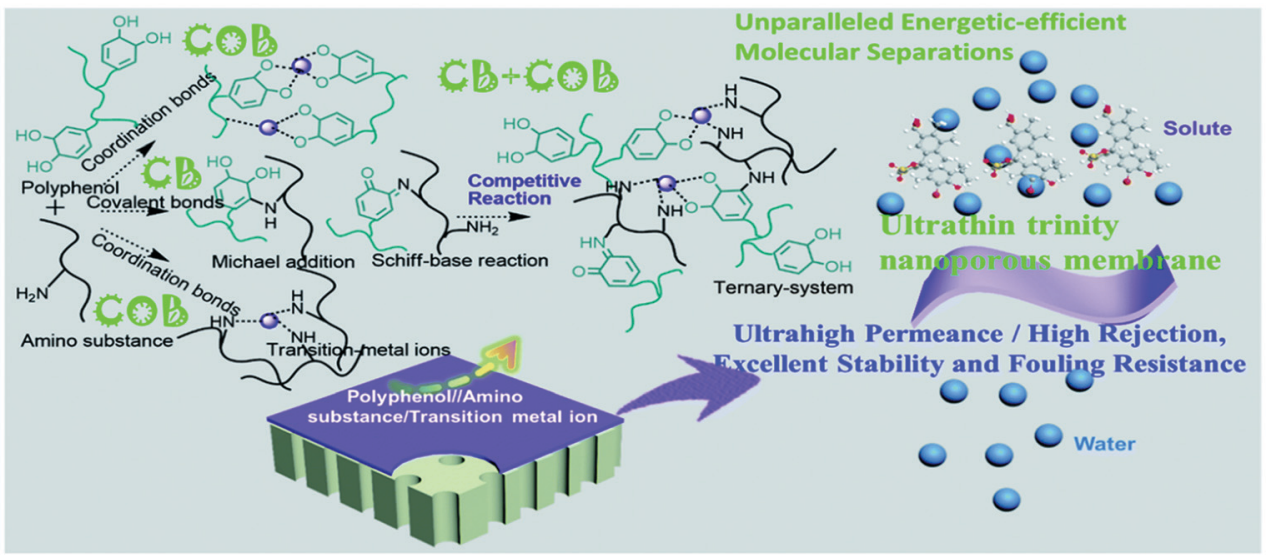

(c)

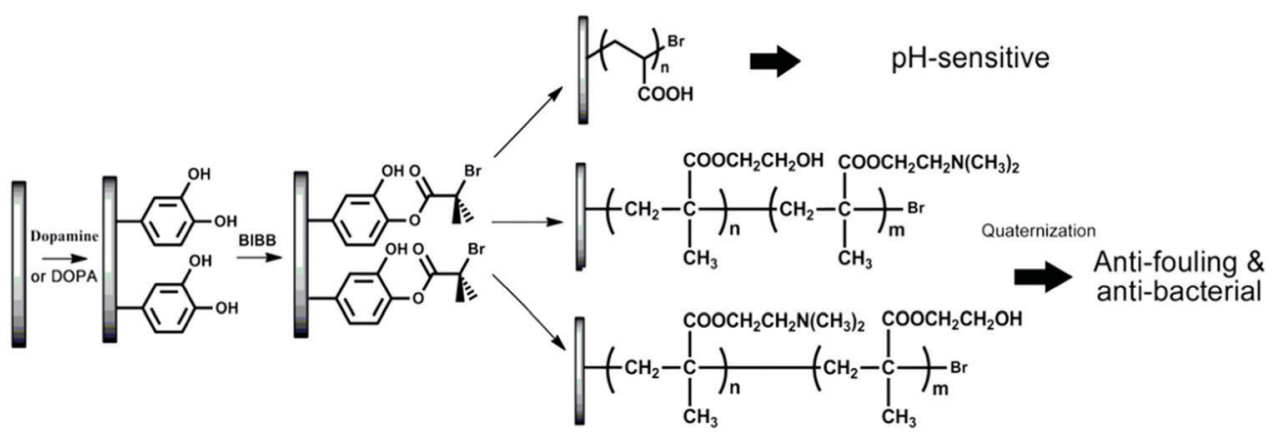

Fig. 5 (a) Illustration for the immobilization of non-ionic polymers on the PVDF membranes by applying mussel-inspired chemistry. Reproduced with permission. ${ }^{97}$ Copyright 2017, Elsevier. (b) The scheme of the fabrication process of the ultrathin trinity coating via covalent bond (CB)/coordination bond (COB) competitive reactions. Reproduced with permission. ${ }^{99}$ Copyright 2020, The Royal Society of Chemistry. (c) Schematic diagram illustrating the process of SI-ATRP from the PVDF membrane for polyacrylic acid (PAA), poly(hydroxyethyl methylacrylate (HEMA)- $g$-dimethylamino ethyl methacrylate (DMAEMA)) and poly(DMAEMA-g-HEMA) grafting. Reproduced with permission. ${ }^{100}$ Copyright 2012, Elsevier.

monomers can react with PEI and $\mathrm{Co}^{2+}$ in the ternary coating system through covalent bonds (PDA/PEI) or coordination bonds $\left(\mathrm{PDA} / \mathrm{Co}^{2+}\right)$ to limit the formation of PDA aggregates, constructing a much thinner and smoother coating layer without blocking the membrane pores, as compared to the conventional PDA deposition methods. The covalent bond (CB)/coordination bond (COB) competitive reactions in the ternary coating system affected the hydrophilicity, thickness and roughness of the membrane surface, facilitating the fabrication of membranes with ultra-high permeance and excellent anti-biofouling ability.

Besides the direct deposition method, dopamine or PDA could also serve as a versatile intermediate layer for post-modification to introduce other functional materials to further modify the
PDA-coated membranes. The membranes are pre-modified with PDA layers, which act as an adherent platform to immobilize both organic and inorganic components such as polymers, oligomers and nanoparticles on the membrane surfaces. Functional polymers are usually grafted onto the surfaces of PDA-coated membranes through surface-initiated polymerization methods. ${ }^{70,100,101}$ For example, the hydrophilic polymer brushes could be covalently anchored onto a PDA-coated membrane by SI-ATRP for constructing functional polymer membranes (Fig. 5c). Jiang et al. grafted poly(sulfobetaine methacrylate) (PSBMA), a commonly used zwitterionic polymer, onto PVDF membranes by PDA-mediated ATRP, which successfully improved the anti-biofouling and antibacterial properties. ${ }^{102}$ 
Similarly, Meng et al. covalently anchored zwitterionic random copolymers consisting of methacryloyloxyethyl phosphorylcholine (MPC) and nitrophenoxycarbonyloxyethyl methacrylate (NPCEMA) monomers on the PVDF micro-filtration membrane through a PDA mediation layer, which exhibited superhydrophilicity as well as excellent anti-fouling performance against oil, bacteria and Bovine Serum Albumin (BSA) protein. ${ }^{103}$ PDA also provides a versatile intermediate layer for depositing inorganic materials for functionalizing membranes due to the excellent metal-ion coordination ability of catechol through bidentate chelation. For instance, silver nanoparticledecorated membranes were prepared by immobilizing silver particles on a PDA-coated membrane via a facile redox reaction owing to the metal-binding ability of phenolic hydroxyl groups in PDA. ${ }^{104-107}$ The Ag/PDA-coated membranes exhibited ultrahigh antibacterial activity, which completely eliminated the attachment of Escherichia coli (E. coli) with a high rejection rate. In addition, graphene oxide (GO) nanosheets were also applied to fabricate nanofiltration (NF) membranes with anti-fouling properties via a PDA intermediate layer. Wang et al. prepared the GO/PDA membrane by pre-modifying a PDA layer on the NF membrane surface, which acted as a strong adhesive agent to bind a layer of GO nanosheets on the surface. ${ }^{108}$ The epoxy and carboxyl groups in GO were able to react with the amine group of PDA, enabling the prepared composite membrane to be chemically and mechanically stable. It was shown that the deposition of PDA and the GO layer enhanced the hydrophilicity and smoothness of the membrane surface, which could significantly mitigate the adsorption of foulants and increase the fouling resistance to BSA. Furthermore, with the assistance of the mussel-inspired versatile platform, the layer-by-layer (LBL) assembly strategy could also be utilized for preparing membranes with excellent anti-biofouling performance and long-term stability. ${ }^{109,110}$ For example, Guo et al. applied polyphenol tannic acid (with abundant catechol groups) and hydrophilic Jeffamine (containing amino groups) in a LBL process to fabricate composite NF membranes, which exhibited excellent resistance against BSA adsorption due to the hydrophilic membrane surface. ${ }^{111}$

3.2.2. Mussel-inspired anti-biofouling coatings. Catechol is able to deposit on both organic and inorganic substrates through diverse chemical interactions, which makes DOPA chemistry (especially PDA) a versatile surface modification strategy to functionalize different surfaces with anti-biofouling properties. On one hand, the hydrophilic PDA coatings can attract water molecules to form a hydration layer that resists foulant adhesion to the substrates. ${ }^{112}$ It was demonstrated that the friction forces of diverse surfaces such as glass, polydimethylsiloxane (PDMS), and epoxy in water were significantly decreased after coated with the PDA layer due to the presence of the hydration layer; thus, the PDA film was expected to serve as a water-based lubrication coating. ${ }^{113}$ Moreover, the surface energy of both hydrophilic and hydrophobic substrates could also be drastically altered after being coated with PDA. For example, the polar component of the surface energy of hydrophobic L-poly(lactic-acid) (PLLA) increased from $9.6 \mathrm{~mJ} \mathrm{~m}^{-2}$ to $47.2 \mathrm{~mJ} \mathrm{~m}^{-2}$ after treatment with dopamine for $14 \mathrm{~h}$ in Tris buffer solution
( $\mathrm{pH}$ 8.5), suggesting that the surface became much more polar and hydrophilic after PDA deposition. ${ }^{114}$ On the other hand, PDA has been found to possess intrinsic antimicrobial activity. Avis and co-workers reported that dopamine self-polymerized onto $E$. coli cells and encapsulated the bacteria in the PDA shells, creating a barrier between $E$. coli and the surroundings. The presence of PDA reduced the permeability of nutrition components for $E$. coli survival and hindered the release of toxic metabolic waste, which prevented further cell multiplication. ${ }^{36}$ Many studies reported that the PDA coating reduced the bacteria proliferation and enhanced antibacterial activity on different types of substrates such as metal, ${ }^{115,116}$ zirconia, ${ }^{117}$ cotton and glass. ${ }^{118}$ However, compared to other antibacterial materials such as metals or polymers, the antibacterial performance of polydopamine was relatively weak. Therefore, researchers combined the PDA with other antibacterial materials to improve the antimicrobial efficacy of coatings. Nanoparticles of various inorganic compounds such as metals, metal oxides and silica have been successfully coated with PDA films on substrates for antibiofouling purposes. For example, Ag NPs were preferentially selected for deposition with PDA to modify the surfaces of diverse materials including graphene, ${ }^{119} \mathrm{Si}$ wafer, ${ }^{120}$ silk fibers, ${ }^{121}$ titanium alloys, ${ }^{122}$ etc. The reducing capability of PDA toward metal ions could ease the oxidization and aggregation of Ag NPs, which enhanced the stability and antibacterial functions against E. coli and Staphylococcus aureus. Magnetite nanoparticles (e.g., $\mathrm{Fe}_{3} \mathrm{O}_{4}{ }^{123-125}$ ) were also incorporated into the PDA polymer matrix to yield a magnetic $\mathrm{Fe}_{3} \mathrm{O}_{4}$ /PDA nanocomposite that was applied for the removal of multiple pollutants (Fig. 6a). The asprepared nanocomposites loaded on different surfaces exhibited excellent biomedical properties, including antiviral, antibacterial and antitumor activities, and could be collected and recovered by using an external magnetic field.

Besides coupling with inorganic materials, the abundant catechol and amine/imine groups in dopamine/PDA molecules could serve as active sites to react with various functional groups of polymers. ${ }^{35,128}$ The synthesis of mussel-inspired functional coatings is generally achieved either by the "grafting from" method by the utilization of mussel-mimetic surface initiators, or the "grafting to" method by employing adhesive anchors based on DOPA chemistry. ${ }^{26,129}$

For the "grafting from" strategy, the substrates are generally functionalized with a PDA layer by an immersion coating process first, where the PDA layer acts as an initiator for the grafting of other antifouling polymers such as PEG or zwitterionic molecules, through diverse interactions related to catechol/ quinone groups. For example, Li et al. used self-polymerized dopamine to form a thin and surface-adherent PDA layer onto the poly(ethylene terephthalate) (PET) sheet, followed by covalent grafting of zwitterionic cysteine that was conjugated with the as-prepared surface via the thiol group through Michael addition. ${ }^{130}$ The results showed that the cysteine immobilized PET surface possessed high resistance to nonspecific protein adsorption and platelet adhesion. Likewise, Ma et al. covalently grafted poly(MPC) brushes onto a PDA-coated titanium (Ti) substrate. The abundant reactive groups $\left(-\mathrm{NH}_{2}\right.$ and $-\mathrm{OH}$ groups $)$ 
(a)

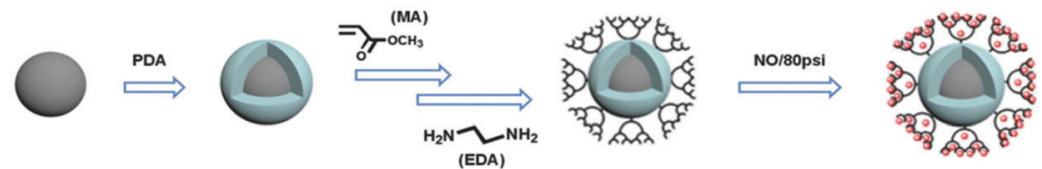

$\mathrm{Fe}_{3} \mathrm{O}_{4}$ Cluster

$\mathrm{Fe}_{3} \mathrm{O}_{4} @ P D A$

$\mathrm{Fe}_{3} \mathrm{O}_{4} @ P D A @ P A M A M-G 3$

$\mathrm{Fe}_{3} \mathrm{O}_{4} @ P D A @ P A M A M @ N O N O a t e$
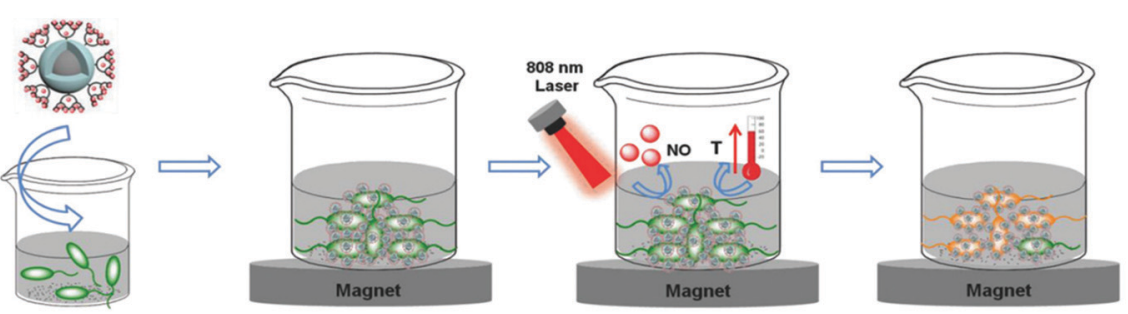

Live bacteria+Materials

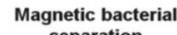
separation

Synergistic antibacterial effects

Dead bacteria

(b)
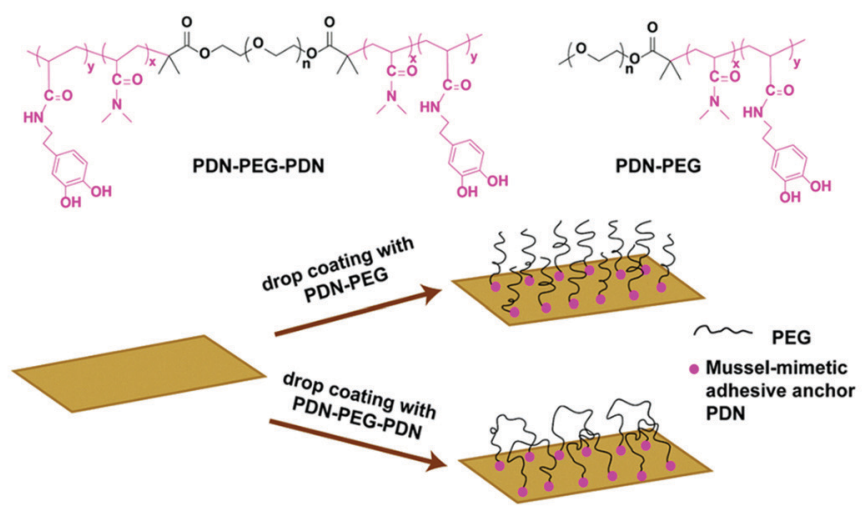

(d)
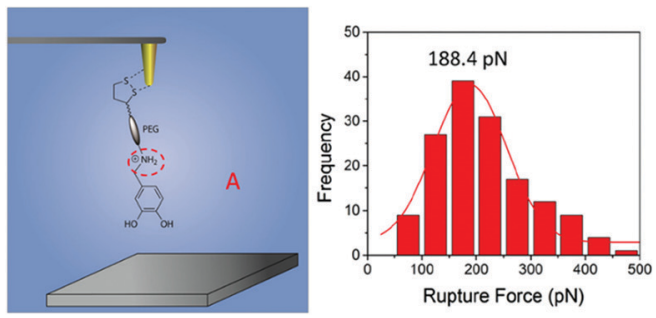
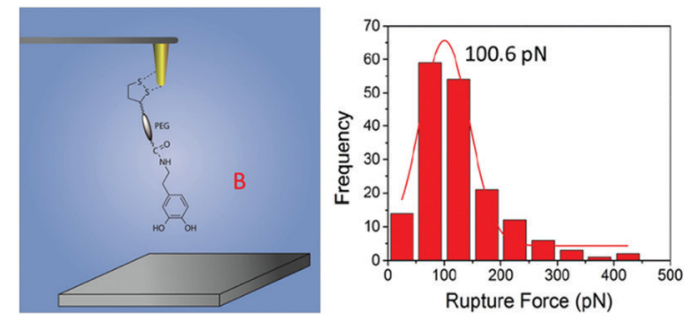

Fig. 6 (a) Synthetic process of $\mathrm{Fe}_{3} \mathrm{O}_{4}$ ( PPAA apoly(amidoamine) $@ \mathrm{~N}$-diazeniumdiolate nanocomposite, and its application for magnetic separation, synergistic photothermal and NO antibacterial effects against both E. coli and S. aureus. Reproduced with permission. ${ }^{125} \mathrm{Copyright} 2018$, Wiley-VCH. (b) Chemical structure of the triblock copolymer PDN-PEG-PDN and the diblock copolymer PDN-PEG and schematics of the preparation of surfaces bearing polymer brushes and polymer loops using the drop coating method. The anti-biofouling performance is shown in (c). The top image shows the changes in frequency and dissipation associated with the adsorption of protein BSA on a bare silica sensor, silica sensors coated with PDN-PEG brushes and PDN-PEG-PDN loops using a QCM-D. The bottom image shows protein adsorption on the three substrate surfaces (ng $\left.\mathrm{cm}^{-2}\right)$ before and after rinsing with PBS buffer. Reproduced with permission. ${ }^{126}$ Copyright 2015, The Royal Society of Chemistry. (d) Schematic illustration of the experimental setup for measuring the interactions between amine-modified catechol ligand A and mica and unmodified catechol B and mica at a single-molecule level, and the correlated force distribution histograms of A-mica and B-mica interactions at the same loading rate $\left(360 \mathrm{nN} \mathrm{s}{ }^{-1}\right)$. Reproduced with permission. ${ }^{127}$ Copyright 2018, American Chemical Society.

of PDA could form an initiator layer to bridge poly (MPC) via SI-ATRP. The mussel-inspired poly (MPC) coating was proved to improve the hemocompatibility of biomaterials and antibiofouling properties. ${ }^{131}$ Likewise, Li et al. covalently grafted zwitterionic poly(MPC) polymer brushes on a variety of PDAcoated materials by surface-initiated activators regenerated by electron transfer atom-transfer radical polymerization (PDA-SIARGET-ATRP). The prepared zwitterionic polymer coatings were demonstrated to have ultralow biofouling performance, which effectively suppressed the adhesion of proteins and platelets. ${ }^{132}$ Due to a high grafting ratio and grafting density, the "grafting from" method allows close control of the architecture of the 
coated polymers, which is capable of producing dense polymer layers. However, this approach usually involves multi-step treatments, leading to increased operation processes and production costs.

The mussel-inspired "grafting to" strategy was pioneered by Messersmith's group in 2003, where the monomethoxyterminated PEG polymer conjugated with a mussel-inspired adhesive catechol ligand was first prepared, and then directly and covalently anchored on gold and titanium surfaces through the adsorption process. ${ }^{32}$ Following this idea, much effort has been dedicated to the preparation of antifouling polymers endtethered mussel-inspired adhesive anchors such as single DOPA residues, which facilitated the polymers to stably bound to the target surfaces. Our group developed an antifouling coating bearing polymer loops using a mussel-inspired ABA triblock copolymer, which showed excellent protein-reduction performance as indicated by the adsorption tests using a quartz crystal microbalance with dissipation (QCM-D) and direct force measurements using SFA. ${ }^{126}$ As shown in Fig. 6b, musselinspired catechol-functionalized poly( $N, N$-dimethylacrylamide) and PEG were selected as the adhesive A block and antifouling B block, respectively. This copolymer was synthesized by reversible additional fragment transfer (RAFT) polymerization and drop-coated on the mica substrate to form a layer consisting of polymer loops with the assistance of the mussel-mimetic adhesive anchors on both ends. The stable anchoring points provided by the catechol groups facilitated the grafting of PEG chains, which achieved a large excluded volume to reduce the protein penetration and exhibited great potential in antifouling applications (Fig. 6c). We also reported a robust and substrateindependent anchoring strategy based on a modified catechol unit with an adjacent cationic amine. The cationic aminemodified catechol ligand showed universal anchoring capability and superior binding stability for tethering anti-biofouling molecules on various substrates. ${ }^{127}$ The interaction mechanisms between the hydrophilic mica surface and catechol with/without cationic amine modification were quantified at the molecular level using single-molecule force spectroscopy based on atomic force microscopy (SM-AFM). As shown in Fig. 6d, the results indicated the enhanced stability of the hydrogen binding between cation amine-modified catechol and mica, suggesting that the synergistic effect of the amine cation and catechol disrupted the hydration layer on mica and facilitated the anchoring of the mussel-inspired ligand on the hydrophilic substrate under water. Due to the ease of operation, the "grafting to" method carries great convenience and feasibility for modifying surfaces with antifouling polymers which were conjugated with stable and applicable surface anchors.

\section{Summary and perspectives}

This work summarizes the catechol-mediated adhesion and cohesion mechanisms for biomaterial synthesis and provides an overview of the recent progress in the design and development of anti-biofouling materials involving hydrogels, membranes, and coatings based on mussel-inspired chemistry. Various bioinspired material fabrication processes and surface functionalization strategies are presented, where materials based on the mussel-inspired chemistry serve as surface modifiers, intermediate layers or adhesive moieties to bind other components for functionalizing materials with anti-biofouling performance. DOPA and its derivatives such as dopamine and PDA have been widely employed in anti-biofouling material fabrication through diverse strategies. Because of the intrinsic hydrophilic and antibacterial properties of catechol and amine groups in DOPA/ dopamine, the catecholamines could be directly deposited as surface modifiers to endow materials with fouling resistance properties. Due to the universality and versatility, materials based on DOPA chemistry could act as adhesive agents to introduce diverse organic/inorganic components, or serve as polymerization initiators/surface anchors to bind functional polymers onto different material surfaces through different DOPA-mediated interactions, constructing mussel-inspired materials with enhanced resistance and inhibition against cells, bacteria and fungi.

Although mussel-inspired strategies hold great promise in the design of anti-biofouling and antibacterial materials for a wide range of applications, there are some challenges remaining in this area. First, the underlying mechanisms of mussel-inspired adhesion chemistry are still not fully understood, which include, but are not limited to, the polymerization mechanism of dopamine, the structure of PDA and the adhesion mechanism of PDA, limiting the capability to replicate and apply the intriguing mussel-inspired chemistry into biomaterial development. Second, the slow deposition kinetics of the PDA film has become an obstacle for surface modification, causing dopamine molecules to polymerize in bulk solution instead of depositing on target surfaces, which may lead to low utilization efficiency of dopamine. The polymerization rate of dopamine could be speeded up by improving the reaction conditions such as using high temperature and vigorous stirring, or adding oxidants such as $\mathrm{Cu}^{2+}$ and $\mathrm{H}_{2} \mathrm{O}_{2} \cdot{ }^{35,133}$ Co-depositing dopamine with polyamines such as PEI can also accelerate the deposition rate of PDA. ${ }^{134}$ Furthermore, the high cost of dopamine still remains a challenge for its widespread practical application. Therefore, facile preparation methods and low-cost alternative chemistries with faster kinetics are highly desired to fabricate mussel-inspired functional materials (e.g., antifouling materials and surfaces). Plant polyphenols with a similar structure to dopamine such as tannic acid, epigallocatechin gallate and epigallocatechin may become promising substitutes of dopamine, while the universality and versatility of the polyphenols need further investigation. Third, PDA molecules mainly exist as oligomers, and the attached PDA layer may not be very stable under certain application conditions, especially in a polar organic solvent, and strong acidic and alkali solutions. ${ }^{132,135,136}$ The stability of PDA layers is closely related to the long-term applications of the immobilized hydrophilic polymer coatings. This review offers an overview of the anti-biofouling materials based on mussel-inspired chemistry, stimulating the development of new strategies and technologies to overcome the long-standing biofouling challenges. Future work 
may focus on further improving the fundamental understanding of the mussel adhesive chemistry and mussel-inspired functionalization strategies. For example, in addition to DOPA chemistry aforementioned, other interactions contributing to mussel adhesion such as metal-catechol coordination, and cation- $\pi$ and anion $-\pi$ interactions also provide alternative strategies for fabricating bio-inspired materials with anti-biofouling applications in various fields.

\section{Conflicts of interest}

There are no conflicts to declare.

\section{Acknowledgements}

We gratefully acknowledge the financial support from the Natural Sciences and Engineering Research Council of Canada (NSERC), the Canada Foundation for Innovation (CFI), and the Canada Research Chairs Program.

\section{References}

1 I. Banerjee, R. C. Pangule and R. S. Kane, Adv. Mater., 2011, 23, 690-718.

2 R. Zhang, Y. Liu, M. He, Y. Su, X. Zhao, M. Elimelech and Z. Jiang, Chem. Soc. Rev., 2016, 45, 5888-5924.

3 H. Vaisocherová, E. Brynda and J. Homola, Anal. Bioanal. Chem., 2015, 407, 3927-3953.

4 A. Mutschler, L. Tallet, M. Rabineau, C. Dollinger, M.-H. Metz-Boutigue, F. Schneider, B. Senger, N. E. Vrana, P. Schaaf and P. Lavalle, Chem. Mater., 2016, 28, 8700-8709.

5 S. B. Darling, J. Appl. Phys., 2018, 124, 30901.

6 D. M. Yebra, S. Kiil and K. Dam-Johansen, Prog. Org. Coat., 2004, 50, 75-104.

7 E. M. Hetrick and M. H. Schoenfisch, Chem. Soc. Rev., 2006, 35, 780-789.

8 N. Barraud, D. J. Hassett, S.-H. Hwang, S. A. Rice, S. Kjelleberg and J. S. Webb, J. Bacteriol., 2006, 188, 7344-7353.

9 R. Kumar, A. M. Isloor, A. F. Ismail, S. A. Rashid and T. Matsuura, RSC Adv., 2013, 3, 7855-7861.

10 B. Peng, J. Wang, Z. Peng, S. Zhou, F. Wang, Y. Ji, Z. Ye, X. Zhou, T. Lin and X. Zhang, Sci. China: Chem., 2012, 55, 435-442.

11 M. Lejars, A. Margaillan and C. Bressy, Chem. Rev., 2012, 112, 4347-4390.

12 C. Yang, X. Ding, R. J. Ono, H. Lee, L. Y. Hsu, Y. W. Tong, J. Hedrick and Y. Y. Yang, Adv. Mater., 2014, 26, 7346-7351.

13 P. Kingshott, J. Wei, D. Bagge-Ravn, N. Gadegaard and L. Gram, Langmuir, 2003, 19, 6912-6921.

14 J. H. Park and Y. H. Bae, Biomaterials, 2002, 23, 1797-1808.

15 S. Jiang and Z. Cao, Adv. Mater., 2010, 22, 920-932.

16 S. Chen and S. Jiang, Adv. Mater., 2008, 20, 335-338.

17 D. Chen, T. Liu, J. Kang, R. Xu, Y. Cao and M. Xiang, Adv. Mater. Interfaces, 2019, 6, 1900706.

18 Q. Shao and S. Jiang, Adv. Mater., 2015, 27, 15-26.
19 P. Zhang, S. Wang, S. Wang and L. Jiang, Small, 2015, 11, 1939-1946.

20 W. Liu and S. Wei, Adv. Mater., 2009, 21, 665-669.

21 T.-S. Wong, S. H. Kang, S. K. Y. Tang, E. J. Smythe, B. D. Hatton, A. Grinthal and J. Aizenberg, Nature, 2011, 477, 443-447.

22 C. Howell, T. L. Vu, C. P. Johnson, X. Hou, O. Ahanotu, J. Alvarenga, D. C. Leslie, O. Uzun, A. Waterhouse and P. Kim, Chem. Mater., 2015, 27, 1792-1800.

23 C. M. Magin, D. L. Alge and K. S. Anseth, Biomed. Mater., 2016, 11, 22001.

24 J. H. Ryu, S. Hong and H. Lee, Acta Biomater., 2015, 27, 101-115.

25 S. Fleischer and T. Dvir, Curr. Opin. Biotechnol., 2013, 24, 664-671.

26 H. Lee, S. M. Dellatore, W. M. Miller and P. B. Messersmith, Science, 2007, 318, 426-430.

27 Y. H. Ding, M. Floren and W. Tan, Biosurfaces, 2016, 2, 121-136.

28 H.-C. Yang, J. Luo, Y. Lv, P. Shen and Z.-K. Xu, J. Membr. Sci., 2015, 483, 42-59.

29 H. Yang, R. Z. Waldman, M. Wu, J. Hou, L. Chen, S. B. Darling and Z. Xu, Adv. Funct. Mater., 2018, 28, 1705327.

30 Z. Wang, H.-C. Yang, F. He, S. Peng, Y. Li, L. Shao and S. B. Darling, Matter, 2019, 1, 115-155.

31 L. Li, PhD thesis, University of Alberta, 2017.

32 J. L. Dalsin, B.-H. Hu, B. P. Lee and P. B. Messersmith, J. Am. Chem. Soc., 2003, 125, 4253-4258.

33 L. Li and H. Zeng, Biotribology, 2016, 5, 44-51.

34 B. D. McCloskey, H. B. Park, H. Ju, B. W. Rowe, D. J. Miller, B. J. Chun, K. Kin and B. D. Freeman, Polymer, 2010, 51, 3472-3485.

35 Y. Liu, K. Ai and L. Lu, Chem. Rev., 2014, 114, 5057-5115.

36 Z. Iqbal, E. P. C. Lai and T. J. Avis, J. Mater. Chem., 2012, 22, 21608-21612.

37 H. Cho, S. K. Madhurakkat Perikamana, J. Lee, J. Lee, K.-M. Lee, C. S. Shin and H. Shin, ACS Appl. Mater. Interfaces, 2014, 6, 11225-11235.

38 X. Liu, J. Cao, H. Li, J. Li, Q. Jin, K. Ren and J. Ji, ACS Nano, 2013, 7, 9384-9395.

39 W.-B. Tsai, W.-T. Chen, H.-W. Chien, W.-H. Kuo and M.-J. Wang, Acta Biomater., 2011, 7, 4187-4194.

40 H. Lee, J. Rho and P. B. Messersmith, Adv. Mater., 2009, 21, 431-434.

41 Q. Lu, E. Danner, J. H. Waite, J. N. Israelachvili, H. Zeng and D. S. Hwang, J. R. Soc., Interface, 2013, 10, 20120759.

42 E. W. Danner, Y. Kan, M. U. Hammer, J. N. Israelachvili and J. H. Waite, Biochemistry, 2012, 51, 6511-6518.

43 N. Bandara, H. Zeng and J. Wu, J. Adhes. Sci. Technol., 2013, 27, 2139-2162.

44 D. S. Hwang, H. Zeng, A. Masic, M. J. Harrington, J. N. Israelachvili and J. H. Waite, J. Biol. Chem., 2010, 285, 25850-25858.

45 J. H. Waite, J. Biol. Chem., 1983, 258, 2911-2915.

46 D. R. Filpula, S. Lee, R. P. Link, S. L. Strausberg and R. L. Strausberg, Biotechnol. Prog., 1990, 6, 171-177. 
47 S. W. Taylor, J. H. Waite, M. M. Ross, J. Shabanowitz and D. F. Hunt, J. Am. Chem. Soc., 1994, 116, 10803-10804.

48 L. M. Rzepecki, K. M. Hansen and J. H. Waite, Biol. Bull., 1992, 183, 123-137.

49 M. A. Even, J. Wang and Z. Chen, Langmuir, 2008, 24, 5795-5801.

50 L. A. Burzio and J. H. Waite, Biochemistry, 2000, 39, 1114711153.

51 J. H. Waite and X. Qin, Biochemistry, 2001, 40, 2887-2893.

52 H. Zhao and J. H. Waite, J. Biol. Chem., 2006, 281, 26150-26158.

53 H. Zhao and J. H. Waite, Biochemistry, 2006, 45, 14223-14231.

54 S. C. Warner and J. H. Waite, Mar. Biol., 1999, 134, 729734.

55 J. Yu, W. Wei, E. Danner, R. K. Ashley, J. N. Israelachvili and J. H. Waite, Nat. Chem. Biol., 2011, 7, 588-590.

56 J. H. Waite and M. L. Tanzer, Science, 1981, 212, 10381040.

57 Q. Lin, D. Gourdon, C. Sun, N. Holten-Andersen, T. H. Anderson, J. H. Waite and J. N. Israelachvili, Proc. Natl. Acad. Sci. U. S. A., 2007, 104, 3782-3786.

58 H. Lee, N. F. Scherer and P. B. Messersmith, Proc. Natl. Acad. Sci. U. S. A., 2006, 103, 12999-13003.

59 S. Kim, J. Huang, Y. Lee, S. Dutta, H. Y. Yoo, Y. M. Jung, Y. Jho, H. Zeng and D. S. Hwang, Proc. Natl. Acad. Sci. U. S. A., 2016, 113, E847-E853.

60 S. Kim, H. Y. Yoo, J. Huang, Y. Lee, S. Park, Y. Park, S. Jin, Y. M. Jung, H. Zeng and D. S. Hwang, ACS Nano, 2017, 11, 6764-6772.

61 C. Lim, J. Huang, S. Kim, H. Lee, H. Zeng and D. S. Hwang, Angew. Chem., Int. Ed., 2016, 55, 3342-3346.

62 Q. Lu, D. S. Hwang, Y. Liu and H. Zeng, Biomaterials, 2012, 33, 1903-1911.

63 Q. Lu, D. X. Oh, Y. Lee, Y. Jho, D. S. Hwang and H. Zeng, Angew. Chem., 2013, 125, 4036-4040.

64 M. Yu, J. Hwang and T. J. Deming, J. Am. Chem. Soc., 1999, 121, 5825-5826.

65 J. Zhang, L. Xiang, B. Yan and H. Zeng, J. Am. Chem. Soc., 2020, 142, 1710-1714.

66 C. A. Monnier, D. G. DeMartini and J. H. Waite, Nat. Commun., 2018, 9, 1-9.

67 N. Holten-Andersen, M. J. Harrington, H. Birkedal, B. P. Lee, P. B. Messersmith, K. Y. C. Lee and J. H. Waite, Proc. Natl. Acad. Sci. U. S. A., 2011, 108, 2651-2655.

68 M. J. LaVoie, B. L. Ostaszewski, A. Weihofen, M. G. Schlossmacher and D. J. Selkoe, Nat. Med., 2005, 11, 1214-1221.

69 X. Fan, L. Lin, J. L. Dalsin and P. B. Messersmith, J. Am. Chem. Soc., 2005, 127, 15843-15847.

70 B. Zhu and S. Edmondson, Polymer, 2011, 52, 2141-2149.

71 H. Ceylan, M. Urel, T. S. Erkal, A. B. Tekinay, A. Dana and M. O. Guler, Adv. Funct. Mater., 2013, 23, 2081-2090.

72 M. Krogsgaard, M. A. Behrens, J. S. Pedersen and H. Birkedal, Biomacromolecules, 2013, 14, 297-301.

73 H. Zeng, D. S. Hwang, J. N. Israelachvili and J. H. Waite, Proc. Natl. Acad. Sci. U. S. A., 2010, 107, 12850-12853.

74 B. Yang, C. Lim, D. S. Hwang and H. J. Cha, Chem. Mater., 2016, 28, 7982-7989.
75 N. Huang, S. Zhang, L. Yang, M. Liu, H. Li, Y. Zhang and S. Yao, ACS Appl. Mater. Interfaces, 2015, 7, 17935-17946.

76 S. Hong, Y. S. Na, S. Choi, I. T. Song, W. Y. Kim and H. Lee, Adv. Funct. Mater., 2012, 22, 4711-4717.

77 D. R. Dreyer, D. J. Miller, B. D. Freeman, D. R. Paul and C. W. Bielawski, Langmuir, 2012, 28, 6428-6435.

78 M. Cencer, Y. Liu, A. Winter, M. Murley, H. Meng and B. P. Lee, Biomacromolecules, 2014, 15, 2861-2869.

79 M. Cencer, M. Murley, Y. Liu and B. P. Lee, Biomacromolecules, 2015, 16, 404-410.

80 H. Meng, P. K. Forooshani, P. U. Joshi, J. Osborne, X. Mi, C. Meingast, R. Pinnaratip, J. Kelley, A. Narkar and W. He, Acta Biomater., 2019, 83, 109-118.

81 C. Liu, W. Yao, M. Tian, J. Wei, Q. Song and W. Qiao, Biomaterials, 2018, 179, 83-95.

82 Y. Xie, Y. Zheng, J. Fan, Y. Wang, L. Yue and N. Zhang, ACS Appl. Mater. Interfaces, 2018, 10, 22692-22702.

83 G. Gao, Y.-W. Jiang, H.-R. Jia and F.-G. Wu, Biomaterials, 2019, 188, 83-95.

84 J. L. Clement and P. S. Jarrett, Met. - Based Drugs, 1994, 1, 467-482.

85 J. Guo, W. Sun, J. P. Kim, X. Lu, Q. Li, M. Lin, O. Mrowczynski, E. B. Rizk, J. Cheng and G. Qian, Acta Biomater., 2018, 72, 35-44.

86 D. E. Fullenkamp, J. G. Rivera, Y. Gong, K. H. A. Lau, L. He, R. Varshney and P. B. Messersmith, Biomaterials, 2012, 33, 3783-3791.

87 L. Li, B. Yan, J. Yang, L. Chen and H. Zeng, Adv. Mater., 2015, 27, 1294-1299.

88 L.-C. Su, Z. Xie, Y. Zhang, K. T. Nguyen and J. Yang, Front. Bioeng. Biotechnol., 2014, 2, 23.

89 J. Guo, W. Wang, J. Hu, D. Xie, E. Gerhard, M. Nisic, D. Shan, G. Qian, S. Zheng and J. Yang, Biomaterials, 2016, 85, 204-217.

90 L. Li, B. Yan, J. Yang, W. Huang, L. Chen and H. Zeng, ACS Appl. Mater. Interfaces, 2017, 9, 9221-9225.

91 Z.-Y. Xi, Y.-Y. Xu, L.-P. Zhu, Y. Wang and B.-K. Zhu, J. Membr. Sci., 2009, 327, 244-253.

92 J. Jiang, L. Zhu, L. Zhu, H. Zhang, B. Zhu and Y. Xu, ACS Appl. Mater. Interfaces, 2013, 5, 12895-12904.

93 B. Yu, J. Liu, S. Liu and F. Zhou, Chem. Commun., 2010, 46, 5900-5902.

94 S. Azari and L. Zou, J. Membr. Sci., 2012, 401, 68-75.

95 Q. Liu, B. Yu, W. Ye and F. Zhou, Macromol. Biosci., 2011, 11, 1227-1234.

96 A. Nguyen, S. Azari and L. Zou, Desalination, 2013, 312, 82-87.

97 Y. He, L. Xu, X. Feng, Y. Zhao and L. Chen, J. Membr. Sci., 2017, 539, 421-431.

98 L. Liu, B. Shao and F. Yang, Sep. Purif. Technol., 2013, 118, 226-233.

99 Y. Zhang, J. Ma and L. Shao, J. Mater. Chem. A, 2020, 8, 5078-5085.

100 Y. Sui, X. Gao, Z. Wang and C. Gao, J. Membr. Sci., 2012, 394, 107-119.

101 C. Zhang, H.-N. Li, Y. Du, M.-Q. Ma and Z.-K. Xu, Langmuir, 2017, 33, 1210-1216. 
102 J. Jiang, P. Zhang, L. Zhu, B. Zhu and Y. Xu, J. Mater. Chem. $B, 2015,3,7698-7706$.

103 F.-N. Meng, M.-Q. Zhang, K. Ding, T. Zhang and Y.-K. Gong, J. Mater. Chem. A, 2018, 6, 3231-3241.

104 H. Ma, P. Gao, Y. Zhang, D. Fan, G. Li, B. Du and Q. Wei, RSC Adv., 2013, 3, 25291-25295.

105 K. P. Wai, C. H. Koo, Y. L. Pang, W. C. Chong and W. J. Lau, J. Water Process Eng., 2020, 33, 100989.

106 H. Yang, Y. Lan, W. Zhu, W. Li, D. Xu, J. Cui, D. Shen and G. Li, J. Mater. Chem., 2012, 22, 16994-17001.

107 L. Huang, S. Zhao, Z. Wang, J. Wu, J. Wang and S. Wang, J. Membr. Sci., 2016, 499, 269-281.

108 C. Wang, M. J. Park, D. H. Seo and H. K. Shon, Sep. Purif. Technol., 2021, 254, 117604.

109 C. Cheng, S. Li, W. Zhao, Q. Wei, S. Nie, S. Sun and C. Zhao, J. Membr. Sci., 2012, 417, 228-236.

110 H. Liu, G. Liu, M. Zhang, H. Zhao, Y. Jiang and J. Gao, Sep. Purif. Technol., 2020, 253, 117519.

111 D. Guo, Y. Xiao, T. Li, Q. Zhou, L. Shen, R. Li, Y. Xu and H. Lin, J. Colloid Interface Sci., 2020, 560, 273-283.

112 E. Li, Z. Du and S. Yuan, Sci. China: Chem., 2013, 56, 773-781.

113 W. Zhang, F. K. Yang, Y. Han, R. Gaikwad, Z. Leonenko and B. Zhao, Biomacromolecules, 2013, 14, 394-405.

114 A. Bourmaud, J. Riviere, A. Le Duigou, G. Raj and C. Baley, Polym. Test., 2009, 28, 668-672.

115 T. S. Sileika, H.-D. Kim, P. Maniak and P. B. Messersmith, ACS Appl. Mater. Interfaces, 2011, 3, 4602-4610.

116 A. Liu, L. Zhao, H. Bai, H. Zhao, X. Xing and G. Shi, ACS Appl. Mater. Interfaces, 2009, 1, 951-955.

117 M. Liu, J. Zhou, Y. Yang, M. Zheng, J. Yang and J. Tan, Colloids Surf., B, 2015, 136, 74-83.

118 L. Su, Y. Yu, Y. Zhao, F. Liang and X. Zhang, Sci. Rep., 2016, 6, 1-8.

119 Z. Zhang, J. Zhang, B. Zhang and J. Tang, Nanoscale, 2013, 5, 118-123.
120 J. Zhang, Y. S. Cheah, S. Santhanakrishnan, K. G. Neoh and C. L. L. Chai, Polymer, 2017, 116, 5-15.

121 Z. Lu, J. Xiao, Y. Wang and M. Meng, J. Colloid Interface Sci., 2015, 452, 8-14.

122 Z. Wang, J. Ou, Y. Wang, M. Xue, F. Wang, B. Pan, C. Li and W. Li, Surf. Coat. Technol., 2015, 280, 378-383.

123 Y. Fang, C. Xing, S. Zhan, M. Zhao, M. Li and H. Liu, J. Mater. Chem. B, 2019, 7, 1933-1944.

124 S. Hemmati, M. M. Zangeneh and A. Zangeneh, Polyhedron, 2020, 177, 114327.

125 S. Yu, G. Li, R. Liu, D. Ma and W. Xue, Adv. Funct. Mater., 2018, 28, 1707440.

126 L. Li, B. Yan, L. Zhang, Y. Tian and H. Zeng, Chem. Commun., 2015, 51, 15780-15783.

127 L. Han, L. Gong, J. Chen, J. Zhang, L. Xiang, L. Zhang, Q. Wang, B. Yan and H. Zeng, ACS Appl. Mater. Interfaces, 2018, 10, 2166-2173.

128 F. Wu, N. Pu, G. Ye, T. Sun, Z. Wang, Y. Song, W. Wang, X. Huo, Y. Lu and J. Chen, Environ. Sci. Technol., 2017, 51, 4606-4614.

129 J. L. Dalsin and P. B. Messersmith, Mater. Today, 2005, 8, 38-46.

130 P. Li, X. Cai, D. Wang, S. Chen, J. Yuan, L. Li and J. Shen, Colloids Surf., B, 2013, 110, 327-332.

131 W. Ma, P. Yang, J. Li, S. Li, P. Li, Y. Zhao and N. Huang, Appl. Surf. Sci., 2015, 349, 445-451.

132 N. Li, T. Li, X.-Y. Qiao, R. Li, Y. Yao and Y.-K. Gong, ACS Appl. Mater. Interfaces, 2020, 12, 12337-12344.

133 P. Zhou, Y. Deng, B. Lyu, R. Zhang, H. Zhang, H. Ma, Y. Lyu and S. Wei, PLoS One, 2014, 9, e113087.

134 W.-Z. Qiu, H.-C. Yang and Z.-K. Xu, Adv. Colloid Interface Sci., 2018, 256, 111-125.

135 S. Kim, T. Gim and S. M. Kang, Prog. Org. Coat., 2014, 77, 1336-1339.

136 A. Belanger, A. Decarmine, S. Jiang, K. Cook and K. A. Amoako, Langmuir, 2018, 35, 1984-1988. 